\title{
Aspectos biopsicossociais de idosos de ambos os sexos participantes de um projeto de extensão para a promoção da saúde
}

\author{
Biopsychosocial aspects of older adults (males and females) participating in an extension project for \\ health promotion
}

Aspectos biopsicosociales de ancianos de ambos sexos participantes en un proyecto de extensión para la promoción de la salud

\author{
Isabella Caroline Santos \\ ORCID: https://orcid.org/0000-0003-4490-6026 \\ Universidade Cesumar, Brasil \\ E-mail: isabellacaroline_@hotmail.com \\ Henrique Broio de Souza \\ ORCID: https://orcid.org/0000-0001-5569-5573 \\ Universidade Cesumar, Brasil \\ E-mail: henriqbroio@gmail.com \\ Marielle Priscila de Paula Silva Lalucci \\ ORCID: https://orcid.org/0000-0003-0671-1893 \\ Universidade Cesumar, Brasil \\ E-mail: mariellepriscila@gmail.com \\ Braulio Henrique Magnani Branco \\ ORCID: https://orcid.org/0000-0002-4625-9128 \\ Universidade Cesumar, Brasil \\ E-mail: braulio.branco@unicesumar.edu.br
}

\begin{abstract}
Resumo
Introdução: Ao mesmo tempo que a população tem vivido mais, às doenças crônicas não-transmissíveis (DCNT's) também tem impactado maior parcela da população idosa. Objetivo: analisar eventuais correlações entre variáveis biopsicossociais de idosos de ambos os sexos e comparar as respostas entre sexos. Métodos: Trata-se de um estudo transversal, realizado em fevereiro/2020, em um projeto de extensão em Maringá/PR, com 30 idosos. Foram analisadas variáveis bioquímicas (aspartato-AST e alanina aminotransferase-ALT, triglicerídeos-TG, colesterol totalCT, lipoproteínas de alta densidade-HDL-c, baixa densidade-LDL-c, muito baixa densidade-VLDL-c, fosfatase alcalina-FA, gama-glutamiltransferase-GGT e glicose em jejum-GLI); antropometria/composição corporal (índice de massa corporal-IMC, massa muscular esquelética-MME, massa de gordura-MG e percentual de gordura-\%G); aptidão física (flexão de antebraço, flexibilidade e caminhada de 6-min) e questionários (Body Shape QuestionnaireBSQ, Autoestima, Ansiedade-EHAA e Qualidade do Sono de Pittsburg-IQSP). As correlações foram interpretadas segundo Hopkins et al. (2009). Resultados: o grupo geral apresentou correlações entre \%G e TG ( $r=0,36-$ efeito moderado); IQSP e EHAA ( $\mathrm{r}=0,44-$-efeito moderado) e IQSP e MG ( $\mathrm{r}=0,39$-efeito moderado) e para o grupo feminino, foram observadas correlações entre \% e GLI ( $\mathrm{r}=0,54$-efeito grande); \% e TG ( $\mathrm{r}=0,64$-efeito grande); \%G e GGT ( $r=0,59-$-efeito grande); \%G e VDL-c ( $r=0,63$-efeito grande) e IQSP e EHAA (r=0,72-efeito muito grande), todas com $\mathrm{p}<0,05$. Não foram identificadas correlações significativas para as demais variáveis $(\mathrm{p}>0,05)$. Conclusão: os resultados apontam distinções entre os sexos, sugerindo a necessidade de adaptações para programas de combate às DCNT's.
\end{abstract}

Palavras-chave: Pessoa de idade; Promoção da saúde; Qualidade de vida.

\begin{abstract}
Introduction: While the population has lived longer, chronic non-communicable diseases (NCD's) has also impacted a larger portion of the elderly population. Objective: To analyze possible correlations between the biopsychosocial variables of elderly, males and females, and compare the responses among them. Methods: This is a cross-sectional study, conducted in February/2020, in an extension project in Maringá/PR, with 30 older adults. Biochemical variables were analyzed (aspartate-AST and alanine aminotransferase-ALT, triglycerides-TG, total cholesterol-TC, high-density lipoproteins-HDL-c, low-density-LDL-c, very low-density-VLDL-c, alkaline phosphatase-AP gammaglutamyltransferase-GGT and fasting glucose-FBGL); Anthropometry/body composition (body mass index-BMI, skeletal muscle mass-SMM, fat mass-FM, and body fat-\%BF); physical fitness (forearm flexion, flexibility, and 6-min walk test) and questionnaires (Body Shape Questionnaire-BSQ, Self-esteem, Anxiety-HAM-A and Sleep Quality of
\end{abstract}


Pittsburg- PSQI). The correlations were interpreted according to Hopkins et al. (2009). Results: The general group presented correlations between \%BF and TG ( $\mathrm{r}=0.36$-moderate effect); PSQI and HAM-A ( $\mathrm{r}=0.44$-moderate effect) and PSQI and FM (r=0.39-moderate effect) and for the female group, it were observed correlations between $\% \mathrm{BF}$ and FBGL ( $\mathrm{r}=0.54-$ large effect); \%BF and TG (r=0.64-large effect); \%BF and GGT ( $\mathrm{r}=0.59-$ large effect); \%BF and VLDL-c ( $\mathrm{r}=0.63$-large effect) and PSQI and HAM-A ( $\mathrm{r}=0.72$-very large effect), all with $\mathrm{p}<0.05$. No significant correlations were identified for the other variables $(\mathrm{p}>0.05)$. Conclusion: The results indicated differences among males and females' elderly adults, suggesting the need for adjustments to programs to combat NCD's.

Keywords: Aged; Health promotion; Quality of life.

\section{Resumen}

Introducción: Al mismo tiempo que la población ha vivido más, las enfermedades crónicas no transmisibles (ECNT's) también han impactado la mayor parte de la población anciana. Objetivo: Analizar eventuales correlaciones entre variables biopsicosociales de ancianos de ambos sexos y comparar las respuestas entre sexos. Métodos: Se trata de un estudio transversal, realizado en febrero/2020, en un proyecto de extensión en Maringá/PR, con 30 ancianos. Se analizaron variables bioquímicas (aspartato-AST y alanina aminotransferasa-ALT, triglicéridos-TG, colesterol totalCT, lipoproteínas de alta densidad-HDL-c, baja densidad-LDL-c, muy baja densidad-VLDL-c, fosfatasa alcalina-FA, gamma-glutamiltransferasa-GGT y glucosa en ayunas-GLI); Antropometría/composición corporal (índice de masa corporal-IMC, masa muscular esquelética-MME, masa grasa-MG y porcentaje de grasa-\%G); aptitud física (flexión de antebrazo, flexibilidad y caminata de 6-min) y cuestionarios (Body Shape Questionnaire-BSQ, Autoestima, Ansiedad-EHAA y Calidad del Sueño de Pittsburg-IQSP). Las correlaciones fueron interpretadas según Hopkins et al. (2009). Resultados: El grupo general presentó correlaciones entre \%G y TG (r=0,36-efecto moderado); IQSP y EHAA ( $\mathrm{r}=0,44$-efecto moderado) e IQSP y MG ( $\mathrm{r}=0,39$-efecto moderado) y para el grupo femenino, fueron observadas correlaciones entre \%G y GLI (r=0,54-efecto grande); \%G y TG (r=0,64-efecto grande); \%G y GGT (r=0,59-efecto grande); \%G y VDL-c ( $r=0,63$-efecto grande) e IQSP y EHAA ( $r=0,72$-efecto muy grande), todas con $\mathrm{P}<0,05$. No se identificaron correlaciones significativas para las demás variables $(\mathrm{p}>0,05)$. Conclusión: Los resultados apuntan distinciones entre los sexos, sugiriendo la necesidad de adaptaciones para programas de combate a las ECNT's.

Palabras clave: Anciano; Calidad de vida; Promoción de la salud.

\section{Introdução}

É considerado idoso, todo indivíduo com idade igual ou superior a 60 anos, no cenário brasileiro (Brasil; Ministério da Saúde, 2013). No ano de 2000, o Instituto Brasileiro de Geografia e Estatística (IBGE) apontou que 1 a cada 10 brasileiros apresentava mais de 60 anos de idade, sendo que a estimativa é de que essa relação será de 1:4 até o ano de 2060 (Instituto Brasileiro de Geografia e Estatística, 2018). A mudança demográfica apresentada está associada ao aumento da expectativa de vida (Rodrigues et al., 2019). Perspectiva essa que cresce proporcionalmente a implantação e execução de programas multidisciplinares de promoção da saúde, que visam estimular o envelhecimento ativo, diminuir a prevalência de doenças crônicas, incentivar o autocuidado e fortalecer redes de apoio (Amaral et al., 2020). Projetos desta natureza, além de melhorar a qualidade de vida dos participantes, contribuem com a manutenção do sistema público de saúde, através de estratégias de menor custo, quando comparadas aos cuidados secundários e terciários como a hospitalização, os tratamentos, a reabilitação e a institucionalização (World Health Organization, 2005).

A partir do início do século XX, com o crescente interesse na saúde do idoso, fez-se necessário voltar a atenção dos profissionais para as alterações fisiológicas, funcionais e estruturais relacionadas ao envelhecimento (Gómez-Gómez \& Zapico, 2019). Entre elas, a modificação da composição corporal, como a redução da estatura e da massa corporal, diminuição da massa livre de gordura (MLG) e alterações nos compartimentos de gordura; os quais nessa faixa etária, são observados menores acúmulos de tecido adiposo periférico, em relação ao tecido adiposo visceral (Moreira et al., 2006). O sedentarismo que tende a acompanhar o processo de envelhecimento, é uma das razões da diminuição da capacidade funcional e da aptidão física entre os idosos, dado que esse é um fator de risco para doenças crônico-degenerativas nessa população, sendo, portanto, indispensável avaliar a capacidade funcional e aptidão física dessas pessoas, a fim de orientar intervenções específicas que evitem esse declínio e prolonguem a independência desses indivíduos aos cuidados de terceiros (Gómez-Gómez \& Zapico, 2019). 
O estado nutricional inadequado também faz parte dos fatores de risco, tanto para doenças crônicas não transmissíveis (DCNT's) - como a diabetes mellitus tipo 2 (DM2) e a hipertensão arterial sistêmica (HAS) - quanto para o número excessivo de óbitos. A primeira é frequentemente associada ao sobrepeso e à obesidade, enquanto a segunda é comum como resultado da desnutrição (Bueno et al., 2008). Estudos mostram que a situação nutricional relatada pode estar associada a diversos fatores, como: a condição socioeconômica, a dificuldade de acesso à informação e alterações nos processos de ingestão, digestão e absorção, frequentemente associadas ao uso de medicamentos (Gilliland et al., 2019).

Atualmente, pesquisas têm demonstrado uma associação positiva entre atividade física e boa saúde mental (Lorés Casanova et al., 2020). Fator que reforça a importância de avaliar também a transformação psicológica ocorrida paralelamente na velhice. Essa mudança pode ser analisada através de fatores como a ansiedade, o humor, a autoestima e a autopercepção da imagem corporal, com o objetivo de que o idoso tenha consciência de sua finitude que, apesar disso, busque uma velhice saudável, sendo ele, o principal responsável pelo seu próprio bem-estar (Oliveira et al., 2019).

Assim, em vista dos fatores elencados, os objetivos do presente estudo foram analisar eventuais correlações e comparações entre variáveis biopsicossociais de idosos. Como justificativa, acredita-se as informações frente aos aspectos biopsicossociais de idosos ingressantes em um projeto de extensão para o combate à obesidade poderão nortear ações mais assertivas para a recuperação das condições de saúde, dessa respectiva população.

\section{Metodologia}

\subsection{Delineamento experimental}

$\mathrm{O}$ presente estudo se caracteriza por apresentar um delineamento transversal e observacional. Foram realizadas correlações e comparações entre às medidas biopsicossociais conduzidas, as quais são apresentadas nas sessões a seguir. Em vista disso, os idosos passaram por uma série de testes, a fim de identificar o perfil bioquímico, antropométrico, de composição corporal, de aptidão física, psicológico e de qualidade do sono.

Participaram do estudo 30 idosos (15 homens e 15 mulheres) com idade igual ou superior a 60 anos, ingressantes de um programa interdisciplinar com o foco em promoção da saúde, realizado pela equipe do Laboratório Interdisciplinar de Intervenção em Promoção da Saúde (LIIPS/UNICESUMAR) localizado em Maringá, no Paraná. Os idosos foram selecionados via amostragem de conveniência, com $\alpha=0,05$ e $\beta=80 \%$. Seguiu-se a recomendação (Jensen et al., 2014) que aponta que uma amostra representativa para análise de estudos com obesidade devem contemplar $\geq 15$ participantes por grupo. Para a realização das avaliações, os idosos deveriam: (a) se inscrever pelo site, por telefone ou pessoalmente para realização das avaliações biopsicossociais; (b) declarar disponibilidade para a participação nas avaliações; (c) ser classificado como apto para a prática de exercícios físicos pela equipe médica do LIIPS/UNICESUMAR e (d) assinar o Termo de Consentimento Livre e Esclarecido (TCLE). O estudo foi aprovado pelo Comitê de Ética em Pesquisa com Seres Humanos da UNICESUMAR, sob o $n^{\circ}$ 2.596.181, sendo todos os procedimentos seguiram as recomendações propostas pela resolução 466/2012 do Ministério da Saúde.

\subsection{Medidas bioquímicas}

Para os exames bioquímicos, as amostras de sangue foram coletadas no laboratório de análises clínicas da universidade, pela manhã, após jejum de aproximadamente 12 horas, durante o mês de fevereiro/2020, seguindo todas as recomendações da Sociedade Brasileira de Patologia Clínica/Medicina Laboratorial (Andriolo et al., 2014). As amostras de sangue foram coletadas em tubo a vácuo Vacuplast ${ }^{\circledR}$, contendo gel e ativador de coágulo para obtenção do soro, sendo centrifugadas a $3.000 \mathrm{rpm}$ por 15 minutos, em temperatura ambiente. Foi utilizado o analisador automático de bioquímica e 
turbidimétrico URIT 8021@ da MHLab para dosagem dos seguintes parâmetros bioquímicos: aspartato aminotransferase (AST), alanina aminotransferase (ALT), glicose em jejum, triglicerídeos (TG), colesterol total (CT), lipoproteínas de alta densidade (HDL-c), lipoproteínas de baixa densidade (LDL-c), fosfatase alcalina e gama-glutamiltransferase (GGT). Para todos os exames de sangue, foram utilizados kits Gold Analisa Diagnóstica (Belo Horizonte, Minas Gerais, Brasil). As análises cumpriram as padronizações especificadas pelo fabricante dos Kits.

Os pontos de corte utilizados para o perfil lipídico foram os propostos pela Sociedade Brasileira de Cardiologia, em que se têm valores desejáveis em jejum de CT $<190 \mathrm{mg} / \mathrm{dL}$, HDL-c $>40 \mathrm{mg} / \mathrm{dL}$, TG $<150 \mathrm{mg} / \mathrm{dL}$, LDL-c <130 mg/dL e nãoHDL-c $<160 \mathrm{mg} / \mathrm{dL}$ (Cavasin, 2016). O valor da lipoproteína de muito baixa densidade (VLDL-c) foi determinado a partir da seguinte equação: VLDL-c $=$ TG/5. Os valores de LDL-c foram estimados a partir da Fórmula de Friedewald (LDL-c $=\mathrm{CT}-$ HDL-c + TG/5) (Cavasin, 2016). Para as enzimas hepáticas os valores de cortes utilizados são os determinados pelo fabricante do kit Gold Analisa Diagnóstica, sendo ALT $<45$ U/L para homens e $<37$ U/L para mulheres, AST $<39$ U/L para homens e < $37 \mathrm{U} / \mathrm{L}$ para mulheres, fosfatase alcalina $<43 \mathrm{U} / \mathrm{L}$ e GGT $<60 \mathrm{U} / \mathrm{L}$ para homens e $<40 \mathrm{U} / \mathrm{L}$ para mulheres (Gold Analisa Diagnóstico, n.d.) e o ponto de corte para glicose em jejum segue as recomendações da Sociedade Brasileira de Diabetes, em que valores $<100 \mathrm{mg} / \mathrm{dL}$ é considerado como glicemia normal (Lyra et al., 2020).

\subsection{Antropometria e composição corporal}

A fim de mensurar a estatura, utilizou-se um estadiômetro fixo (Sanny® Standard, São Paulo, Brasil) de acordo com a padronização estabelecida (Heyward, 2001a). Para a avaliação da composição corporal, utilizou-se uma bioimpedância elétrica multifrequencial tetrapolar de oito pontos táteis (InBody570®, Biospace, Seul, Coreia do Sul). Os idosos foram instruídos acerca dos procedimentos técnicos para obtenção fidedigna das medidas, as quais foram: (1) jejum de quatro horas (sem ingestão de qualquer tipo de alimento sólido ou líquido); (2) urinar anteriormente à medida; (3) não praticar qualquer tipo de atividade física moderada ou intensa, 24 horas antes da medida; (4) não consumir bebidas alcoólicas ou com cafeína, 24 horas antes da medida; (5) não utilizar medicação diurética, 24 horas antes da medida (Branco et al., 2018).

\subsection{Aptidão física relacionada à saúde:}

Flexões máximas de antebraço: para a avaliação das flexões máximas de antebraço, foi utilizado o teste proposto (Rikli \& Jones, 2001). Para o teste, os avaliados permaneceram sentados em uma cadeira com encosto e sem braços, tendo as suas pernas levemente afastadas, com os pés apoiados no chão. Desta forma, os avaliados estavam com os seus braços dominantes estendidos ao lado da cadeira, perpendicularmente ao solo, segurando um halter de $2 \mathrm{~kg}$ para mulheres e $4 \mathrm{~kg}$ para homens. Dessa forma, os idosos foram estimulados a executar o máximo de flexões de antebraço durante 30 segundos.

Flexibilidade no banco de Wells: a fim de avaliar a flexibilidade, foi utilizado o teste sentar e alcançar (Wells \& Dillon, 1952). Para tanto, utilizou-se o Banco de Wells, com o objetivo de identificar a flexibilidade da cadeia posterior. Para a execução do teste, os avaliados permaneceram sentados com o tronco ereto, as duas pernas levemente afastadas e estendidas, com a sola dos pés apoiada no suporte abaixo da bandeja do banco. Após o posicionamento, os idosos foram orientados a colocar os dedos médios um sobre o outro e apoiá-los na régua da bandeja do banco. Desse modo, os participantes foram instruídos a realizar uma inspiração profunda e no momento da expiração, deveriam empurrar a régua o mais distante possível, de forma constante, sem flexionar os joelhos.

Teste de caminhada de 6 minutos: com o intuito de identificar a resistência aeróbia, realizou-se r o teste de caminhada de 6 minutos (TC6) (Rikli \& Jones, 2001). Os idosos deveriam caminhar o mais rápido possível, sem correr, ao redor de um retângulo de 50 metros de comprimento. Os participantes foram instruídos a manter a intensidade de esforço elevada durante toda a duração do teste, isto é, durante 6 minutos. Ao final desse período, foi mensurada a distância percorrida ao final do teste. 


\subsection{Questionários psicológicos e de qualidade do sono}

Questionário de imagem corporal: para a avaliação da imagem corporal, utilizou-se o Body Shape Questionnaire (BSQ), (Cooper et al., 1987), que foi traduzido para o Português (Cordás \& Castilho, 1994) e validado para a população brasileira (Manetta, 2002). O BSQ trata-se de um questionário em escalas tipo likert, com respostas entre 1 até 6 , sendo que o número 1 representa "nunca" e o número 6, "sempre". O BSQ apresenta 34 questões acerca da preocupação com medidas corporais e insatisfação corporal. A insatisfação corporal é dada pelo maior escore, em que os indivíduos são classificados como satisfeitos com sua respectiva imagem corporal, com valores entre 81 até 110 pontos e insatisfeitos, com valores $\geq$ de 111 pontos.

Questionário de autoestima: para a avaliação da autoestima, aplicou-se a Escala de Autoestima validada para o português (Hutz \& Zanon, 2011). O instrumento consiste em 10 afirmações sobre um conjunto de sentimentos relacionados à autoestima e autoaceitação. Trata-se de um questionário em escalas tipo likert, com quatro opções de resposta, sendo elas: "concordo totalmente", "concordo", "discordo" e "discordo totalmente".

Questionário de ansiedade: para a avaliação da ansiedade, utilizou-se a Escala de Hamilton para Avaliação da Ansiedade (EHAA) (Hamilton, 1959) e validada em português (Ito \& Ramos, 1998). A EHAA apresenta 14 grupos de sintomas, subdivididos em dois grupos, sendo sete relacionados aos sintomas de humor ansioso e sete relacionados aos sintomas físicos de ansiedade. Cada item é avaliado segundo uma escala tipo likert de zero a quatro, sendo que o número 0 indica: "sintomas ausentes"; o número 1 indica "sintomas com intensidades médias a baixa"; o número 2 indica "sintomas com intensidades médias a alta"; o número 3 indica "sintomas com intensidade forte"; e o número 4 indica "sintomas com intensidade incapacitante". A soma dos escores obtidos em cada item resulta em um escore total entre 0 até 56 pontos.

Questionário de qualidade do sono: para avaliar a qualidade do sono, utilizou-se o índice de qualidade do sono de Pittsburg (IQSP) (Buysse et al., 1989). O IQSP consiste em 19 questões elaboradas para o avaliado e 5 questões para o seu companheiro de quarto. O IQSP busca avaliar componentes relacionados à qualidade relativa do sono, latência do sono, duração do sono, eficiência habitual do sono, transtornos do sono, uso de medicamentos para dormir e disfunção diurna. A pontuação dos componentes do IQSP é somada gerando um escore global entre 0 até 21 pontos. Um valor de escore global > a 5, sugere que o indivíduo apresenta dificuldades em alguns componentes, ou moderadas em vários.

\subsection{Análise estatística}

Os dados foram tabulados no software Microsoft Excel® (versão 2016, Microsoft, Estados Unidos da América). Utilizou-se o software SPSS® (versão 20.0, IBM, Estados Unidos da América), a fim de calcular as médias, desvios padrões, medianas, valores mínimos e máximos. A normalidade dos dados foi testada via teste de Shapiro-Wilk. Após a confirmação da normalidade $(p>0,05)$, utilizou-se a correlação de Pearson, a fim de verificar possíveis associações entre as variáveis biopsicossociais do presente estudo, assim como o teste te Student para amostras independentes, com o propósito de comparar eventuais diferenças entre as mesmas variáveis, conforme o sexo. Para todos os testes estatísticos foi estabelecido um nível de significância de 5\%. Ressalta-se que foram realizadas correlações gerais, com ambos os sexos e correlações das variáveis apenas considerando o sexo masculino, bem como para o sexo feminino. Para a interpretação das correlações aplicou-se a classificação proposta por Hopkins (Hopkins et al., 2009), sendo: 0,0 até <0,1 (efeito trivial); 0,1 até <0,3 (efeito pequeno); 0,3 até <0,5 (efeito moderado); 0,5 até <0,7 (efeito grande); 0,7 até <0,9 (efeito muito grande); 0,9 até 1 (efeito quase perfeito). 


\section{Resultados}

Os idosos apresentaram uma média de idade correspondente a 67,7 $\pm 4,4$ anos, no grupo geral e entre os sexos os homens obtiveram média de 68,9 $\pm 4,5$ anos de idade e as

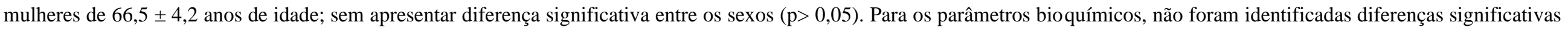

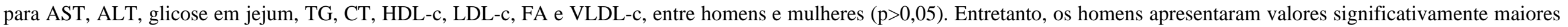
para a GGT, quando comparado às mulheres $(\mathrm{p}<0,05)$ (Tabela 1$)$.

Tabela 1. Comparação entre as medidas bioquímicas de idosos e idosas participantes de um projeto de extensão comunitária no município de Maringá, em fevereiro de 2020.

\begin{tabular}{|c|c|c|c|c|c|c|c|}
\hline & \multicolumn{2}{|c|}{ Geral } & \multicolumn{2}{|c|}{ Masculino } & \multicolumn{2}{|c|}{ Feminino } & \multirow{2}{*}{$\begin{array}{c}\text { Teste } \mathrm{t} \\
\text { Valor de } p\end{array}$} \\
\hline & Média e DP & $\mathrm{IC}$ & Média e DP & $\mathrm{IC}$ & Média & $\mathrm{IC}$ & \\
\hline AST (U/L) & $26,6 \pm 5,2$ & $15-39$ & $27,9 \pm 5,6$ & $19-39$ & $35,3 \pm 4,6$ & $15-33$ & $p=0,18$ \\
\hline ALT (U/L) & $33,0 \pm 11,8$ & $20-75$ & $36,5 \pm 15$ & $21-75$ & $29,5 \pm 6,2$ & $20-49$ & $p=0,10$ \\
\hline Glicose (mg/dL) & $104,2 \pm 27,2$ & $79-231$ & $112,9 \pm 35,3$ & $89-231$ & $95,5 \pm 11,0$ & $79-121$ & $p=0,08$ \\
\hline $\mathrm{TG}(\mathrm{mg} / \mathrm{dL})$ & $107,6 \pm 50,8$ & $79-231$ & $114,5 \pm 56$ & $36-211$ & $100,7 \pm 46,0$ & $37-212$ & $p=0,46$ \\
\hline CT (mg/dL) & $173,7 \pm 29,6$ & $128-227$ & $171,5 \pm 26,5$ & $136-226$ & $175,9 \pm 33,1$ & $128-227$ & $p=0,68$ \\
\hline HDL-c (mg/dL) & $21,2 \pm 6,7$ & $9-36$ & $21,4 \pm 6,8$ & $9-36$ & $21,1 \pm 6,7$ & $12-36$ & $p=0,89$ \\
\hline LDL-c (mg/dL) & $130,9 \pm 33,8$ & $73-196$ & $127,0 \pm 29,6$ & $75-182$ & $134,8 \pm 38,2$ & $73-196$ & $p=0,53$ \\
\hline $\mathrm{FA}(\mathrm{U} / \mathrm{L})$ & $75,7 \pm 19,6$ & $43-109$ & $72,9 \pm 20,5$ & 43-109 & $78,5 \pm 18,9$ & $46-109$ & $p=0,44$ \\
\hline GGT (U/L) & $28,6 \pm 17,2$ & $11-87$ & $35,9 \pm 20,0$ & $15-87$ & $21,3 \pm 9,9$ & $11-51$ & $p=0,01^{*}$ \\
\hline VLDL-c (mg/dL) & $21,5 \pm 10,2$ & $9-36$ & $22,9 \pm 11,2$ & $7-42$ & $20,0 \pm 9,2$ & $7-42$ & $p=0,44$ \\
\hline
\end{tabular}

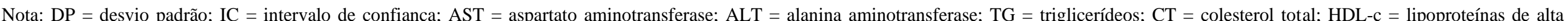

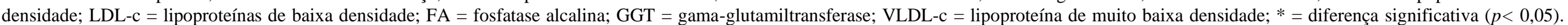
Fonte: Autores.

Para as variáveis de aptidão física relacionada à saúde, não foram verificadas diferenças significativas para o IMC, MME, flexões máximas de antebraço e flexibilidade, entre

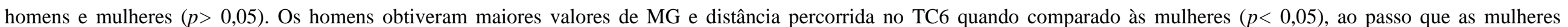




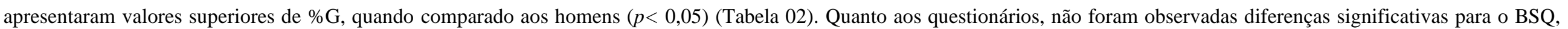

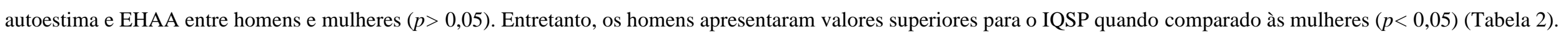

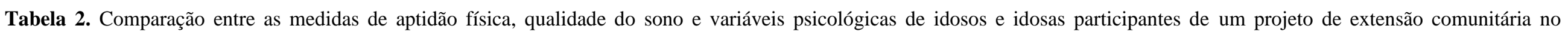
município de Maringá, em fevereiro de 2020.

\begin{tabular}{|c|c|c|c|c|c|c|c|}
\hline & \multicolumn{2}{|c|}{ Geral } & \multicolumn{2}{|c|}{ Masculino } & \multicolumn{2}{|c|}{ Feminino } & \multirow{2}{*}{$\begin{array}{c}\text { Teste } \mathrm{t} \\
\text { Valor de } p\end{array}$} \\
\hline & Média e DP & $\mathrm{IC}$ & Média e DP & $\mathrm{IC}$ & Média e DP & $\mathrm{IC}$ & \\
\hline \multicolumn{8}{|c|}{ Antropometria e composição corporal } \\
\hline IMC $\left(\mathrm{kg} / \mathrm{m}^{2}\right)$ & $29,5 \pm 6,2$ & $20-46$ & $30,1 \pm 5,5$ & $20-42$ & $28,9 \pm 6,9$ & $22-46$ & $p=0,58$ \\
\hline MME (kg) & $31,5 \pm 9,7$ & $14-53$ & $32,5 \pm 7,4$ & $21-50$ & $30,5 \pm 11,8$ & $14-53$ & $p=0,58$ \\
\hline MG (kg) & $26,6 \pm 9,5$ & $11-53$ & $30,2 \pm 12,0$ & $11-53$ & $23,0 \pm 3,8$ & $18-31$ & $p=0,03^{*}$ \\
\hline$\% \mathrm{G}$ & $36,33 \pm 8,0$ & $20-54$ & $32,7 \pm 6,1$ & $20-43$ & $40,0 \pm 8,8$ & $24-54$ & $p=0,01 *$ \\
\hline \multicolumn{8}{|c|}{ Testes de aptidão física } \\
\hline FMA (reps) & $18,5 \pm 4,6$ & $9-26$ & $18,3 \pm 5,0$ & $9-25$ & $18,7 \pm 4,4$ & $12-26$ & $p=0,82$ \\
\hline Flexibilidade $(\mathrm{cm})$ & $24,8 \pm 8,7$ & $10-38$ & $24,3 \pm 9,2$ & $10-38$ & $25,2 \pm 8,4$ & $10-38$ & $p=0,79$ \\
\hline TC6 (mts) & $607,8 \pm 84,3$ & $461-773$ & $648,2 \pm 75,2$ & $512-773$ & $567,5 \pm 75,6$ & $461-670$ & $p=0,00^{*}$ \\
\hline \multicolumn{8}{|c|}{ Questionários } \\
\hline BSQ (pts) & $64,6 \pm 27,6$ & $34-158$ & $57,1 \pm 20,2$ & $34-107$ & $72 \pm 32,4$ & $42-158$ & $p=0,14$ \\
\hline $\mathrm{EA}(\mathrm{pts})$ & $26,3 \pm 3,2$ & $21-33$ & $27 \pm 2,9$ & $22-32$ & $25,6 \pm 3,4$ & $21-33$ & $p=0,23$ \\
\hline EHAA (pts) & $7,6 \pm 5,7$ & $0-19$ & $7,1 \pm 5,2$ & $0-17$ & $8,1 \pm 6,4$ & $0-19$ & $p=0,62$ \\
\hline IQSP (pts) & $10,7 \pm 3,1$ & $6-19$ & $12,5 \pm 2,7$ & $9-19$ & $8,9 \pm 2,4$ & $6-13$ & $p=0,00^{*}$ \\
\hline
\end{tabular}

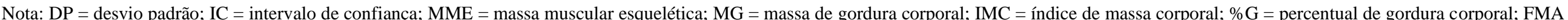

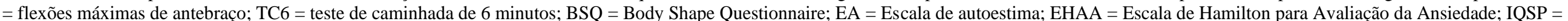
índice de qualidade do sono de Pittsburg; * = diferença significativa $(p<0,05)$. Fonte: Autores. 
Em relação às correlações testadas entre $\% \mathrm{G}$ e os parâmetros bioquímicos, destaca-se a correlação encontrada no grupo total entre \%G e TG $(r=0,36$; $p<0,04 ;$ efeito

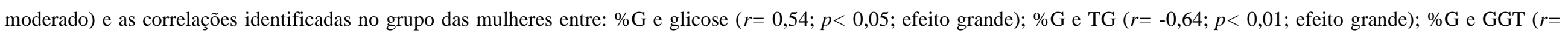
0,59; $p<0,05$; efeito grande) e \%G e VDL-c ( $r=0,63 ; p<0,01$; efeito grande) (Tabela 3$)$.

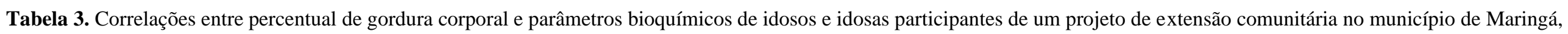
em fevereiro de 2020.

\begin{tabular}{|c|c|c|c|}
\hline Correlações testadas & Coeficiente de correlação & Magnitude do efeito & Valor de $p$ \\
\hline \multicolumn{4}{|c|}{ Geral } \\
\hline$\% \mathrm{Ge}$ AST & $r=-0,02$ & Trivial & $p=0,90$ \\
\hline$\% \mathrm{G} \mathrm{e} \mathrm{ALT}$ & $r=0,06$ & Trivial & $p=0,75$ \\
\hline$\%$ G e Glicose & $r=0,07$ & Trivial & $p=0,71$ \\
\hline$\% \mathrm{G}$ e $\mathrm{TG}$ & $r=0,36$ & Moderado & $p=0,04 *$ \\
\hline$\% \mathrm{G}$ e $\mathrm{CT}$ & $\mathrm{r}=0,02$ & Trivial & $p=0,91$ \\
\hline$\% \mathrm{G}$ e HDL-c & $r=-0,04$ & Trivial & $p=0,84$ \\
\hline$\% \mathrm{G}$ e LDL-c & $r=-0,00$ & Trivial & $p=0,99$ \\
\hline$\%$ G e FA & $r=0,36$ & Moderado & $p=0,05$ \\
\hline$\%$ G e GGT & $r=0,09$ & Trivial & $p=0,64$ \\
\hline$\% \mathrm{G}$ e VDL-c & $r=0,35$ & Moderado & $p=0,6$ \\
\hline \multicolumn{4}{|c|}{ Masculino } \\
\hline$\% \mathrm{Ge}$ AST & $r=0,32$ & Moderado & $p=0,25$ \\
\hline$\% \mathrm{G}$ e ALT & $r=0,44$ & Moderado & $p=0,09$ \\
\hline$\%$ G e Glicose & $r=0,06$ & Trivial & $p=0,82$ \\
\hline$\% \mathrm{G}$ e $\mathrm{TG}$ & $r=0,41$ & Moderado & $p=0,12$ \\
\hline$\% \mathrm{G}$ e CT & $r=-0,09$ & Trivial & $p=0,76$ \\
\hline$\% \mathrm{G}$ e HDL-c & $r=-0,19$ & Pequeno & $p=0,48$ \\
\hline$\% \mathrm{G}$ e LDL-c & $r=-0,09$ & Trivial & $p=0,75$ \\
\hline$\%$ G e FA & $\mathrm{r}=0,43$ & Moderado & $p=0,11$ \\
\hline$\% \mathrm{G}$ e GGT & $\mathrm{r}=0,17$ & Реqueno & $p=0,55$ \\
\hline$\% \mathrm{G}$ e VLDL-c & $\mathrm{r}=0,38$ & Moderado & $p=0,16$ \\
\hline
\end{tabular}


Research, Society and Development, v. 10, n. 12, e74101220116, 2021

(CC BY 4.0) | ISSN 2525-3409 | DOI: http://dx.doi.org/10.33448/rsd-v10i12.20116

\begin{tabular}{|c|c|c|c|}
\hline \multicolumn{4}{|c|}{ Feminino } \\
\hline$\%$ Ge AST & $r=-0,15$ & Pequeno & $p=0,59$ \\
\hline$\% \mathrm{G}$ e ALT & $r=-0,01$ & Trivial & $p=0,98$ \\
\hline$\% \mathrm{G}$ e Glicose & $r=0,54$ & Grande & $p=0,03^{*}$ \\
\hline$\% \mathrm{G}$ e $\mathrm{TG}$ & $r=0,64$ & Grande & $p=0,00^{*}$ \\
\hline$\% \mathrm{G}$ e CT & $r=0,02$ & Trivial & $p=0,94$ \\
\hline$\%$ G e HDL-c & $\mathrm{r}=0,24$ & Pequeno & $p=0,38$ \\
\hline$\%$ G e LDL-c & $r=-0,04$ & Trivial & $p=0,88$ \\
\hline$\%$ G e FA & $r=0,31$ & Moderado & $p=0,26$ \\
\hline$\%$ G e GGT & $r=0,59$ & Grande & $p=0,02 *$ \\
\hline$\% \mathrm{G}$ e VLDL-c & $r=0,63$ & Grande & $p=0,01 *$ \\
\hline
\end{tabular}

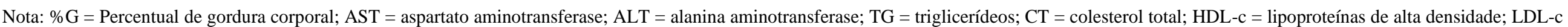

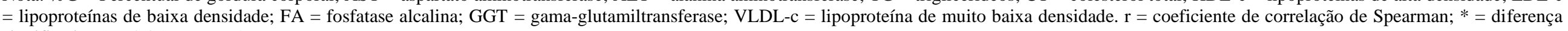
significativa $(\mathrm{p}<0,05)$. Fonte: Autores

Quanto às correlações entre o IQSP e a composição corporal, salienta-se a correlação observada no grupo geral entre o IQSP e MG ( $r=0,39 ; p<0,05$; efeito moderado) (Tabela

4). 
Research, Society and Development, v. 10, n. 12, e74101220116, 2021

(CC BY 4.0) | ISSN 2525-3409 | DOI: http://dx.doi.org/10.33448/rsd-v10i12.20116

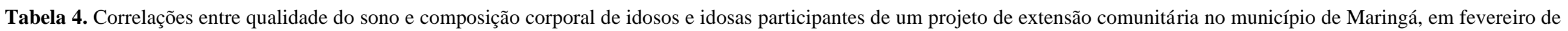
2020 .

\begin{tabular}{|c|c|c|c|}
\hline Correlações testadas & Coeficiente de correlação & Magnitude do efeito & Valor de $p$ \\
\hline \multicolumn{4}{|c|}{ Geral } \\
\hline IQSP e IMC & $r=0,14$ & Pequeno & $p=0,45$ \\
\hline IQSP e MME & $r=-0,05$ & Trivial & $p=0,77$ \\
\hline IQSP e MG & $r=0,39$ & Moderado & $p=0,03^{*}$ \\
\hline IQSP e \%G & $r=-0,21$ & Pequeno & $p=0,26$ \\
\hline \multicolumn{4}{|c|}{ Masculino } \\
\hline IQSP e IMC & $r=0,23$ & Pequeno & $p=0,41$ \\
\hline IQSP e MME & $r=0,03$ & Trivial & $p=0,93$ \\
\hline IQSP e MG & $r=0,16$ & Pequeno & $p=0,57$ \\
\hline IQSP e \%G & $r=0,40$ & Moderado & $p=0,14$ \\
\hline \multicolumn{4}{|c|}{ Feminino } \\
\hline IQSP e IMC & $r=-0,13$ & Pequeno & $p=0,65$ \\
\hline IQSP e MME & $r=-0,24$ & Реquепо & $p=0,38$ \\
\hline IQSP e MG & $r=0,34$ & Moderado & $p=0,22$ \\
\hline IQSP e \%G & $r=-0,16$ & Pequeno & $p=0,56$ \\
\hline
\end{tabular}

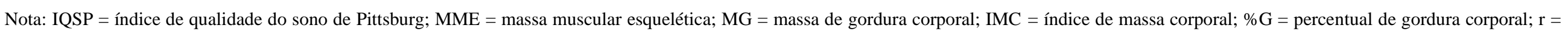
coeficiente de correlação de Spearman; * = diferença significativa $(p<0,05)$. Fonte: Autores.

Com relação às correlações entre O IQSP e as variáveis psicológicas, enfatiza-se a correlação observada no grupo geral entre IQSP e EHAA ( $r=0,44 ; p<0,05$; efeito moderado) e a correlação identificada no grupo das mulheres entre IQSP e EHAA ( $r=0,72 ; p<0,01$; efeito muito grande) (Tabela 5). 
Research, Society and Development, v. 10, n. 12, e74101220116, 2021

(CC BY 4.0) | ISSN 2525-3409 | DOI: http://dx.doi.org/10.33448/rsd-v10i12.20116

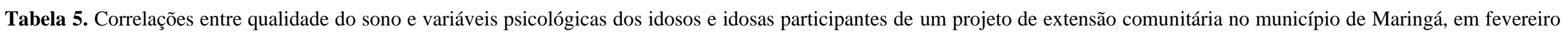
de 2020 .

\begin{tabular}{|c|c|c|c|}
\hline Correlações testadas & Coeficiente de correlação & Magnitude do efeito & Valor de $p$ \\
\hline \multicolumn{4}{|c|}{ Geral } \\
\hline IQSP - BSQ & $r=-0,15$ & Pequeno & $p=0,42$ \\
\hline IQSP - EA & $r=0,28$ & Pequeno & $p=0,13$ \\
\hline IQSP - EHAA & $r=0,44$ & Moderado & $p=0,02 *$ \\
\hline \multicolumn{4}{|c|}{ Masculino } \\
\hline IQSP - BSQ & $r=0,22$ & Pequeno & $p=0,43$ \\
\hline IQSP - EA & $r=0,00$ & Trivial & $p=0,98$ \\
\hline IQSP - EHAA & $r=0,43$ & Moderado & $p=0,11$ \\
\hline \multicolumn{4}{|c|}{ Feminino } \\
\hline IQSP - BSQ & $r=-0,36$ & Moderado & $p=0,19$ \\
\hline IQSP - EA & $r=0,22$ & Pequeno & $p=0,42$ \\
\hline IQSP - EHAA & $r=0,72$ & Muito Grande & $p=0,00 *$ \\
\hline
\end{tabular}

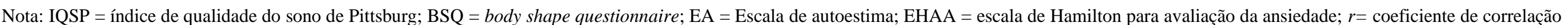
de Spearman; * = diferença significativa $(p<0,05)$. Fonte: Autores. 


\section{Discussão}

A partir da análise dos resultados, observa-se que, apesar do envelhecimento ser um processo natural, a população idosa apresenta um perfil com características particulares a sua respectiva faixa etária. As alterações do estado nutricional, das variáveis bioquímicas e da capacidade física podem definitivamente comprometer a qualidade de vida desses indivíduos. Da mesma forma, observou-se que alguns parâmetros (\%G, GGT, MG e IQSP), de uma maneira geral, apresentaram resultados que merecem ser avaliados, considerando as individualidades de cada sexo.

$\mathrm{Na}$ avaliação do perfil hepático foram observados valores similares de AST, ALT e FA, entre os sexos. Quando as mesmas variáveis foram analisadas separadamente, os homens apresentaram uma média de GGT significativamente mais elevada em comparação com as mulheres. Em vista disso, resultados semelhantes foram encontrados por Valença e colaboradores, na qual valores de AST (feminino: 24,7 \pm 8,5 U/L e masculino: $24 \pm$ 9,4 U/L) e ALT (feminino: 11,8 \pm 6,8 U/L e masculino: $15 \pm 21,5 \mathrm{U} / \mathrm{L}$ ) foram similares entre os sexos, e média de GGT elevada entre os homens, em comparação com as mulheres, sendo masculino: 87,5 \pm 115 U/L e feminino: $55 \pm$ 48,7 U/L (Valença et al., 2018). Estudos apontam uma correlação entre níveis elevados de GGT com aumento do IMC, sugerindo que o estado nutricional (classificação de obesidade) pode ser um dos principais fatores para o aumento sérico dos níveis das enzimas hepáticas (Chielle \& Rocha, 2015). A amostra masculina avaliada apresentou elevação nos níveis da GGT e IMC superior a $30 \mathrm{~kg} / \mathrm{m}^{2}$, fator que relaciona a obesidade com o dano hepático.

$\mathrm{Na}$ avaliação dos participantes do presente estudo, foram observadas correlações positivas entre o \%G e o TG. Ao estratificar os pacientes por sexo, as mulheres apresentaram correlação significativa entre o \%G com a glicose, TG, GGT e VLDL-c. Alterações nos exames bioquímicos, como a glicose em jejum e perfil lipídico podem ser comuns em idosos, ao passo que modificações no estilo de vida podem reverter esse quadro (Buyken et al., 2010). A pesquisa realizada por (Cassiano et al., 2020) verificou 16 semanas de atividade física reduziram os valores médios de CT e LDL-c, aumentando os níveis de HDL-c. Em vista disso, a prática de atividade física regular por idosos é indispensável para manutenção da saúde e qualidade de vida dessa população (Gómez-Gómez \& Zapico, 2019).

Os participantes do presente estudo apresentaram aumento nos níveis de glicose em jejum e perfil lipídico quando comparado aos valores de referência, com exceção do HDL-c que apresentou níveis significativamente reduzidos (Gold Analisa Diagnóstico; Heyward, 2001b; Lyra et al., 2020). Um grande estudo brasileiro envolvendo 49 mil indivíduos mostrou que a prevalência de diabetes e dislipidemias em indivíduos com obesidade é três vezes maior quando comparado a indivíduos dentro dos padrões de normalidade para o IMC (Gigante et al., 2009). Consequentemente, tornam-se indispensáveis propostas de programas para perda e manutenção de peso, a fim de diminuir os índices de obesidade e simultaneamente das comorbidades associadas.

Em relação a MG, os homens apresentaram valores maiores quando comparado às mulheres, sendo estes resultados similares aos obtidos por Alvero-Cruz e colaboradores (Alvero-Cruz et al., 2017). Esse mesmo estudo constatou que idosos do sexo masculino possuem maior quantidade de gordura visceral, quando comparado a indivíduos do sexo feminino; evidências sólidas apontam que o tecido adiposo visceral é um fator intimamente relacionado ao risco para desenvolvimento da a síndrome metabólica e até de vários tipos de cânceres (Alvero-Cruz et al., 2017). Entretanto, com o fim da idade reprodutiva, as mulheres tendem a acumular mais gordura, principalmente na região abdominal devido a diminuição da concentração de estrogênio como resultado do declínio da funcionalidade dos ovários, concomitante ao aumento da secreção de hormônios androgênicos (Dmitruk et al., 2018). Assim, homens e mulheres de diferentes faixas etárias apresentam risco de adiposidade central, tornando indispensáveis intervenções nutricionais e de atividade física, com o propósito de combater às DCNT’s e proporcionar a longevidade saudável. 
Ao analisar a maior distância percorrida no TC6 dos homens quando comparados às mulheres, percebe-se uma similaridade com outros achados (Steffen et al., 2002), em que diferentes faixas etárias, i.e., entre os 60 aos 89 anos de idade; os homens apresentaram média superior ao valor obtido pelas mulheres.

Em relação ao IQSP, os homens apresentaram piores resultados quando comparado às mulheres. Corroboram para este achado, um estudo que constatou que a eficiência do sono, ou seja, a relação entre o tempo total dormindo e o tempo despendido na cama - diminuiu com a idade em ambos os sexos. Já o momento de sono profundo - conhecido como sono de ondas lentas diminuiu com a idade, sendo que os indivíduos do sexo masculino foram os mais afetados (Luca et al., 2015). A correlação entre a qualidade do sono e massa gorda foi anteriormente analisada em um estudo que concluiu que a má qualidade do sono está relacionada ao aumento do tecido adiposo visceral (Sweatt et al., 2018). Entretanto, os resultados encontrados no presente estudo devem ser tratados com cautela, pois essa associação foi encontrada apenas no grupo geral, ignorando as comparaçães por gênero. Sugere-se, portanto, a realização de estudos longitudinais, com a finalidade de testar a relação entre causa e efeito, com o objetivo de confirmar ou refutar nossos achados frente a adiposidade e qualidade do sono entre os sexos.

Ao avaliar o nível de ansiedade dos participantes, observou-se que o grupo geral apresentou uma correlação significativa entre a qualidade do sono e a ansiedade; porém no grupo feminino, o efeito foi mais expressivo ( $r=0,72 p<0,01$; efeito muito grande). Os achados de um estudo recente (Goldstein-Piekarski et al., 2018) apontaram que a ansiedade aumentada em mulheres se deve pelo fato delas serem mais suscetíveis às consequências emocionais da privação e da má qualidade do sono. Ainda em relação à variável da ansiedade, estudos apontam que essa é uma condição psiquiátrica comum em indivíduos da terceira idade (El-Gabalawy et al., 2013).

Os resultados mostram que a população idosa não deve ser tratada como um grupo geral, dado que as variáveis apresentaram diferenças representativas quando comparadas entre os sexos. Com isso, observa-se a importância desses indivíduos receberem atenção básica - tanto no Sistema Único de Saúde (SUS) quanto na rede privada, a fim de considerar a particularidade de cada sexo. Possibilitando, dessa forma, a criação de programas aperfeiçoados e com respostas ainda mais efetivas nessa população de acordo com as suas respectivas especificidades e necessidades. A atenção primária tem papel fundamental na promoção da saúde por meio de ações que orientem os idosos quanto à importância do desenvolvimento constante de suas capacidades funcionais, por meio do emprego de hábitos alimentares saudáveis, prática de exercícios físicos e autocuidado. É papel da equipe multiprofissional informar, orientar, apresentar alternativas e assistir essa população na mudança de comportamento através de medidas educativas e processos informativos eficientes que têm como objetivo final prevenir o acometimento de DCNT's, em especial a obesidade e consequentemente, conter gastos excessivos com tratamentos.

As limitações da pesquisa incluem a ausência da consideração de dados socioeconômicos dos idosos participantes do projeto. Portanto, a generalização dos resultados para outras realidades sociais, econômicas e culturais deve ser cautelosa. Estudos futuros devem considerar essa variável para definição mais ampla do perfil desses participantes, possibilitando assim, a programação e implementação de estratégias de promoção da saúde mais efetivas para essa população.

\section{Conclusão}

Observa-se que a população idosa vem crescendo a cada dia no Brasil, um fenômeno, conhecido por "feminização do envelhecimento" é embasado no fato de mulheres apresentarem maior longevidade em relação ao sexo masculino ${ }^{43}$. Entretanto, nos resultados do presente estudo elas apresentaram índices como: maior percentual de gordura, menor desempenho no teste físico e correlações positivas entre qualidade do sono e maior ansiedade. Indicadores esses que quando em dissonância com a normalidade, comprometem a qualidade de vida dessas mulheres. 
Por sua vez, os homens também apresentaram resultados que implicam na piora da qualidade de vida e da longevidade, como: maior quantidade de massa gorda, pior qualidade do sono e GGT elevada - sendo esse último parâmetro relacionado a uma enzima que quando aumentada indica um possível comprometimento de órgãos como pâncreas, coração e fígado.

De acordo com o exposto, é possível concluir que os indivíduos participantes do estudo apresentaram comprometimento de aspectos relevantes para a qualidade de vida e manutenção da saúde, sendo cada um dos sexos com suas particularidades. Essas diferenças apontam para a necessidade do desenvolvimento de estratégias e ações específicas para cada sexo, com o objetivo de melhorar hábitos e consequentemente aumentar a expectativa de vida dessa população.

\section{Agradecimentos}

À Fundação Araucária de Apoio ao Desenvolvimento Científico e Tecnológico do Paraná que financiou a presente pesquisa.

\section{Referências}

Alvero-Cruz, J. R., Fernández Vázquez, R., García Vega, M. del M., García Lavigne, J. A., Rodríguez Linares, M. V., \& Martínez Blanco, J. (2017). Sensibilidad y especificidad de la adiposidad abdominal con el síndrome metabólico en ancianos. Revista Española de Geriatría y Gerontología, 52(3), 128134. https://doi.org/10.1016/j.regg.2016.11.001

Amaral, M. do, Souza, J. M. M., Luis, Z. dos S., Stofel, N. S., Silva, G. F. M. da, \& Macedo, J. L. De. (2020). Envelhecimento e saúde: promoção de um grupo de idosos na estratégia de saúde da família. Anais Do Salão Internacional de Ensino, Pesquisa e Extensão, 10(3).

Andriolo, A., Faulhaber, A. C. L., Junior, A. P., Martins, A. rodrigues, \& Machado, A. M. de O. (2014). Coleta e preparo da amostra biológica. In Barueri, SP : Manole: Minha Editora (Vol. 4, Issue 8).

Branco, B. H. M., Bernuci, M. P., Marques, D. C., Carvalho, I. Z., Barrero, C. A. L., Oliveira, F. M. de, Ladeia, G. F., \& Júnior, N. N. (2018). Proposal of a normative table for body fat percentages of Brazilian young adults through bioimpedanciometry. Journal of Exercise Rehabilitation, 14(6), 974-979. https://doi.org/10.12965/jer.1836400.200

Brasil; Ministério da Saúde. (2013). Estatuto do Idoso - 3a edição. In 2013. www.saude.gov.br/editora

Bueno, J. M., Martino, H. S. D., Fernandes, M. F. S., Costa, L. S., \& Silva, R. R. (2008). Avaliação nutricional e prevalência de doenças crônicas não transmissíveis em idosos pertencentes a um programa assistencial. Ciência \& Saúde Coletiva, 13(4), 1237-1246. https://doi.org/10.1590/S141381232008000400020

Buyken, A. E., Flood, V., Rochtchina, E., Nestel, P., Brand-Miller, J., \& Mitchell, P. (2010). Modifications in Dietary Fat Quality Are Associated with Changes in Serum Lipids of Older Adults Independently of Lipid Medication. The Journal of Nutrition, 140(1), 88-94. https://doi.org/10.3945/jn.109.110486

Buysse, D. J., Reynolds, C. F., Monk, T. H., Berman, S. R., \& Kupfer, D. J. (1989). The Pittsburgh sleep quality index: A new instrument for psychiatric practice and research. Psychiatry Research, 28(2), 193-213. https://doi.org/10.1016/0165-1781(89)90047-4

Cassiano, A. do N., Silva, T. S. da, Nascimento, C. Q. do, Wanderley, E. M., Prado, E. S., Santos, T. M. de M., Mello, C. S., \& Barros-Neto, J. A. (2020). Efeitos do exercício físico sobre o risco cardiovascular e qualidade de vida em idosos hipertensos. Ciência \& Saúde Coletiva, $25(6), 2203-2212$. https://doi.org/10.1590/1413-81232020256.27832018

Cavasin, S. B. P. (2016). $7^{\text {a }}$ Diretriz Brasileira de Hipertensão Arterial. Sociedade Brasileira de Cardiologia.

Chielle, E., \& Rocha, A. (2015). Avaliação do perfil hepático na obesidade adulto-jovem. Unoesc \& Ciência. 6(1): 15 - 22, $2015.6(1), 15-22$.

Cooper, P. J., Taylor, M. J., Cooper, Z., \& Fairbum, C. G. (1987). The development and validation of the body shape questionnaire. International Journal of Eating Disorders, 6(4), 485-494. https://doi.org/10.1002/1098-108X(198707)6:4<485::AID-EAT2260060405>3.0.CO;2-O

Cordás, T. A., \& Castilho S. (1994). Imagem corporal nos transtornos alimentares - instrumentos de avaliação: "Body Shape Questionnaire.” Psiquiatria Biológica, 2(1), 17-21.

Dmitruk, A., Czeczelewski, J., Czeczelewska, E., Golach, J., \& Parnicka, U. (n.d.). Body composition and fatty tissue distribution in women with various menstrual status. Roczniki Panstwowego Zakladu Higieny, 69(1), 95-101. http://www.ncbi.nlm.nih.gov/pubmed/29519121

El-Gabalawy, R., Mackenzie, C. S., Thibodeau, M. A., Asmundson, G. J. G., \& Sareen, J. (2013). Health anxiety disorders in older adults: Conceptualizing complex conditions in late life. Clinical Psychology Review, 33(8), 1096-1105. https://doi.org/10.1016/j.cpr.2013.08.010

Gigante, D. P., Moura, E. C. de, \& Sardinha, L. M. V. (2009). Prevalência de excesso de peso e obesidade e fatores associados, Brasil, 2006. Revista de Saúde Pública, 43(suppl 2), 83-89. https://doi.org/10.1590/S0034-89102009000900011 
Gilliland, J. A., Shah, T. I., Clark, A., Sibbald, S., \& Seabrook, J. A. (2019). A geospatial approach to understanding inequalities in accessibility to primary care among vulnerable populations. PLOS ONE, 14(1), e0210113. https://doi.org/10.1371/journal.pone.0210113

Gold Analisa Diagnóstico. (n.d.). Instruções de uso dos reagentes. http://www.goldanalisa.com.br/produtos.asp

Goldstein-Piekarski, A. N., Greer, S. M., Saletin, J. M., Harvey, A. G., Williams, L. M., \& Walker, M. P. (2018). Sex, Sleep Deprivation, and the Anxious Brain. Journal of Cognitive Neuroscience, 30(4), 565-578. https://doi.org/10.1162/jocn_a_01225

Gómez-Gómez, M. E., \& Zapico, S. C. (2019). Frailty, Cognitive Decline, Neurodegenerative Diseases and Nutrition Interventions. International Journal of Molecular Sciences, 20(11), 2842. https://doi.org/10.3390/ijms20112842

HAMILTON, M. (1959). THE ASSESSMENT OF ANXIETY STATES BY RATING. British Journal of Medical Psychology, 32(1), 50-55. https://doi.org/10.1111/j.2044-8341.1959.tb00467.x

Heyward, V. (2001a). ASEP methods recommendation: Body composition assessment. Journal of Exercise Physiology Online, 4(4), 1-12.

Heyward, V. (2001b). ASEP methods recommendation: Body composition assessment. In Journal of Exercise Physiology Online.

Hopkins, W. G., Marshall, S. W., Batterham, A. M., \& Hanin, J. (2009). Progressive Statistics for Studies in Sports Medicine and Exercise Science. Medicine \& Science in Sports \& Exercise, 41(1), 3-12. https://doi.org/10.1249/MSS.0b013e31818cb278

Hutz, C. S., \& Zanon, C. (2011). Revisão da adaptação, validação e normatização da Escala de Autoestima de Rosenberg. Aval. Psicol, 10(1), 41-49.

Instituto Brasileiro de Geografia e Estatística. (2018). Projeção da população do Brasil e das Unidades da Federação. https://www.ibge.gov.br/apps/populacao/projecao/index.html

Ito, L. M., \& Ramos, R. T. (1998). Escalas de avaliacao clinica: transtorno de panico / Clinical rating scales: panic disorder. Rev. Psiquiatr. Clín, 25(6), 294302 .

Jensen, M. D., Ryan, D. H., Apovian, C. M., Ard, J. D., Comuzzie, A. G., Donato, K. A., Hu, F. B., Hubbard, V. S., Jakicic, J. M., Kushner, R. F., Loria, C. M., Millen, B. E., Nonas, C. A., Pi-Sunyer, F. X., Stevens, J., Stevens, V. J., Wadden, T. A., Wolfe, B. M., \& Yanovski, S. Z. (2014). 2013 AHA/ACC/TOS Guideline for the Management of Overweight and Obesity in Adults. Circulation, 129(25_suppl_2). https://doi.org/10.1161/01.cir.0000437739.71477.ee

Luca, G., Haba Rubio, J., Andries, D., Tobback, N., Vollenweider, P., Waeber, G., Marques Vidal, P., Preisig, M., Heinzer, R., \& Tafti, M. (2015). Age and gender variations of sleep in subjects without sleep disorders. Annals of Medicine, 47(6), 482-491. https://doi.org/10.3109/07853890.2015.1074271

Lyra, R., Oliveira, M., Lins, D., Cavalcanti, N., Gross, J. L., Maia, F. F. R., Araújo, L. R., Yafi, M., Guimarães, F. P. D. M., Takayanagui, A. M. M., Lucena, J. B. D. S., Golbert, A., Campos, M. A. a., Saúde, M. da, Sartorelli, D. S., Franco, L. J., Prevenção, E., \& Marcondes, J. a. M. (2020). Sociedade Brasileira de Diabetes. In Diabetes Mellitus Tipo 1 e Tipo2 (Vol. 5, Issue 3).

Manetta, M. C. DP. (2002). Validade interna, dimensionalidade e desempenho da escala BSQ “Body Shape Questionnaire” em uma população de estudantes universitários.

Moreira, A. D. J., Nicastro, H., \& Cordeiro, R. C. (2006). Composição corporal de idosos. 8(1), 201-214.

Oliveira, A., Nossa, P., \& Mota-Pinto, A. (2019). Assessing Functional Capacity and Factors Determining Functional Decline in the Elderly: A CrossSectional Study. Acta Médica Portuguesa, 32(10), 654. https://doi.org/10.20344/amp.11974

Roberta E. Rikli, \& C. Jessie Jones. (2001). Senior fitness test manual. Human Kinetics.

Rodrigues, M. F., Farias, N., Vieira De Souza, P. H., Érica, V., \& Santos, S. (2019). O Novo Retrato Demográfico Do Brasil: Análise Acerca Do Envelhecimento Populacional E Suas Decorrências Econômicas the New Demographic Portrait of Brazil: Analysis About Population Aging and Its Economic Decorrices. 7(03), 1-11. https://www.gvaa.com.br/ver

S. Lorés Casanova, J. Rodríguez Mañas, T. Lorente Aznar, C. Pelegrín Valero, A. Castillón Fantova, \& I. Marques Lopes. (2020). La actividad física y el estado nutricional y psicosocial del anciano no dependiente. Semergen Revista Española de Medicina de Familia, 19(4), 244-253.

Steffen, T. M., Hacker, T. A., \& Mollinger, L. (2002). Age- and Gender-Related Test Performance in Community-Dwelling Elderly People: Six-Minute Walk Test, Berg Balance Scale, Timed Up \&amp; Go Test, and Gait Speeds. Physical Therapy, 82(2), 128-137. https://doi.org/10.1093/ptj/82.2.128

Sweatt, S. K., Gower, B. A., Chieh, A. Y., Liu, Y., \& Li, L. (2018). Sleep quality is differentially related to adiposity in adults. Psychoneuroendocrinology, 98, 46-51. https://doi.org/10.1016/j.psyneuen.2018.07.024

Valença, T. V. R., Sangiovo, A., Pereira, F., Vincensi, C., Lissarassa, Y. P. S., Zimmermann, C. E., Comparsi, B., Casalini, C. E. C., Viera, E. K., \& Frizzo, M. N. (2018). Obesidade, diabetes e hipertensão associados a dislipidemia e dano hepático. Revista Saúde Integrada, 11(22), 2-18.

Wells, K. F., \& Dillon, E. K. (1952). The Sit and Reach-A Test of Back and Leg Flexibility. Research Quarterly. American Association for Health, Physical Education and Recreation, 23(1), 115-118. https://doi.org/10.1080/10671188.1952.10761965

World Health Organization. (2005). Envelhecimento ativo: uma política de saúde. 62. 